\title{
Impact of Coronary Dominance on In-Hospital Outcomes after Percutaneous Coronary Intervention in Patients with Acute Coronary Syndrome
}

\author{
Toshiki Kuno $^{1 *}$, Yohei Numasawa ${ }^{1}$, Hiroaki Miyata ${ }^{2}$, Toshiyuki Takahashi ${ }^{1}$, Koichiro Sueyoshi $^{3}$, \\ Takahiro Ohki ${ }^{4}$, Koji Negishi ${ }^{5}$, Akio Kawamura ${ }^{6}$, Shun Kohsaka ${ }^{6}$, Keiichi Fukuda ${ }^{6}$
}

1 Department of Cardiology, Ashikaga Red Cross Hospital, Tochigi, Japan, 2 University of Tokyo, Healthcare Quality Assessment, Tokyo, Japan, 3 Department of Cardiology, Kawasaki City Municipal Hospital, Kanagawa, Japan, 4 Department of Cardiology, Tokyo Dental College Ichikawa General Hospital, Chiba, Japan, 5 Department of Cardiology, Yokohama Municipal Hospital, Kanagawa, Japan, 6 Department of Cardiology, Keio University School of Medicine, Tokyo, Japan

\begin{abstract}
Objective: This study evaluated the manner in which coronary dominance affects in-hospital outcomes of acute coronary syndrome (ACS) patients undergoing percutaneous coronary intervention ( $\mathrm{PCI})$.

Background: Previous studies have shown that left dominant coronary anatomies are associated with worse prognoses in patients with coronary artery disease.

Methods: Data were analyzed from 4873 ACS patients undergoing PCI between September 2008 and April 2013 at 14 hospitals participating in the Japanese Cardiovascular Database Registry. The patients were grouped based on diagnostic coronary angiograms performed prior to $\mathrm{PCl}$; those with right- or co-dominant anatomy (RD group) and those with leftdominant anatomy (LD group).

Results: The average patient age was $67.6 \pm 11.8$ years and both patient groups had similar ages, coronary risk factors, comorbidities, and prior histories. The numbers of patients presenting with symptoms of heart failure, cardiogenic shock, or cardiopulmonary arrest were significantly higher in the LD group than in the RD group (heart failure: $650 \mathrm{RD}$ patients [14.7\%] vs. 87 LD patients [18.8\%], $P=0.025$; cardiogenic shock: 322 RD patients [7.3\%] vs. 48 LD patients [10.3\%], $P=0.021$; and cardiopulmonary arrest: $197 \mathrm{RD}$ patients [4.5\%] vs. 36 LD patients [7.8\%], $P=0.003$ ). In-hospital mortality was significantly higher among LD patients than among RD patients (182 RD patients [4.1\%] vs. 36 LD patients [7.8\%], $P=0.001$ ). Multivariate logistic regression analysis revealed that LD anatomy was an independent predictor for in-hospital mortality (odds ratio, 1.75; 95\% confidence interval, 1.06-2.89; $P=0.030$ ).
\end{abstract}

Conclusion: Among ACS patients who underwent PCI, LD patients had significantly worse in-hospital outcomes compared with RD patients, and LD anatomy was an independent predictor of in-hospital mortality.

Citation: Kuno T, Numasawa Y, Miyata H, Takahashi T, Sueyoshi K, et al. (2013) Impact of Coronary Dominance on In-Hospital Outcomes after Percutaneous Coronary Intervention in Patients with Acute Coronary Syndrome. PLoS ONE 8(8): e72672. doi:10.1371/journal.pone.0072672

Editor: Gerard Pasterkamp, University Medical Center Utrecht, Netherlands

Received September 14, 2012; Accepted July 11, 2013; Published August 26, 2013

Copyright: (c) 2013 Kuno et al. This is an open-access article distributed under the terms of the Creative Commons Attribution License, which permits unrestricted use, distribution, and reproduction in any medium, provided the original author and source are credited.

Funding: The authors have no support or funding to report.

Competing Interests: The authors have declared that no competing interests exist.

* E-mail: kuno-toshiki@hotmail.co.jp

\section{Introduction}

Variations in the balance of the coronary arteries are common, particularly with regard to the supply of the posterior aspect of the left ventricle. In the majority of patients, the right coronary artery (RCA) reaches the crux of the heart and supplies the posterior descending artery (PDA) [1]. Left-dominant (LD) anatomy, described as a variant of normal anatomy, has a prevalence of approximately $5-12 \%$ in the general population [2-5]. In these individuals, the left circumflex artery (LCX) reaches the crux and supplies the posterior descending and, usually, the atrioventricular nodal branches [2].

LD anatomy is believed to be associated with worse prognoses for patients with acute coronary syndrome (ACS) and stable coronary artery disease $[3,6]$. The PDA, arising from the RCA, may serve as a back-up supply in normal anatomy, with the rightdominant (RD) system acting in an overall protective manner. LD patients, in contrast, usually have only the left coronary artery to supply the majority of the myocardium. Thus, an event in a major vessel may, therefore, lead to a worse outcome. Furthermore, technical challenges exist in interventions associated with the LCX artery because of the steepness of its take-off, relative to other arteries [7]. However, data describing the effects of coronary dominance in modern percutaneous coronary intervention (PCI) are scarce. 


\section{Methods}

\section{Study Design}

The Japan Cardiovascular Database (JCD) is a large, ongoing, prospective, multicenter, cohort study designed to collect clinical background and outcome data on PCI patients. Data pertaining to approximately 150 variables are being collected. In this registry, participating hospitals have been instructed to record data from hospital visits for consecutive PCI patients and to register these data into an internet-based database. The database system performs checks to ensure that the reported data are complete and internally consistent. PCIs performed using any commercially available coronary device may be included. The decision to perform PCI is made according to the attending physicians' clinical assessments of the patient. The study does not mandate specific interventional or surgical techniques, such as vascular access, or the use of a specific stent or closure device.

Table 1. Baseline clinical characteristics of each group.

\begin{tabular}{|c|c|c|c|}
\hline & $\begin{array}{l}\text { RD+Co \% } \\
(n=4409)\end{array}$ & $\begin{array}{l}\text { LD \% } \\
(n=464)\end{array}$ & $P$ Value \\
\hline Age $\geq 80$ years & $16.2(714)$ & $18.3(85)$ & 0.236 \\
\hline Age $70-79$ years & $32.3(1422)$ & $32.8(152)$ & 0.835 \\
\hline Female & 22.4 (989) & $19.8(92)$ & 0.217 \\
\hline $\mathrm{BMI} \geq 30$ & $5.4(240)$ & $4.7(22)$ & 0.589 \\
\hline \multicolumn{4}{|l|}{ Coronary risk factors } \\
\hline DM & $37.2(1642)$ & 37.7 (175) & 0.840 \\
\hline DM with insulin & $6.7(297)$ & $8.4(39)$ & 0.178 \\
\hline Hypertension & 70.1 (3089) & $69.0(320)$ & 0.632 \\
\hline Hyperlipidemia & $61.2(2699)$ & $58.6(272)$ & 0.293 \\
\hline Smoking & $39.2(1730)$ & $41.8(194)$ & 0.294 \\
\hline \multicolumn{4}{|l|}{ Comorbidities } \\
\hline CVD & $8.5(375)$ & $9.3(43)$ & 0.601 \\
\hline COPD & $2.7(120)$ & $4.3(20)$ & 0.057 \\
\hline CKD stage $\geq 3$ & $17.5(770)$ & $18.5(86)$ & 0.564 \\
\hline Hemodialysis & $3.8(166)$ & $2.8(13)$ & 0.363 \\
\hline PAD & $5.6(248)$ & $6.3(29)$ & 0.598 \\
\hline \multicolumn{4}{|l|}{ History } \\
\hline Prior MI & $16.3(718)$ & $15.1(70)$ & 0.551 \\
\hline Prior HF & $6.5(286)$ & $7.1(33)$ & 0.621 \\
\hline Prior PCl & $20.6(910)$ & $19.6(91)$ & 0.629 \\
\hline Prior CABG & $4.1(179)$ & $2.4(11)$ & 0.077 \\
\hline \multicolumn{4}{|l|}{ Presenting status } \\
\hline CCS class 4 angina & $15.4(681)$ & $16.6(77)$ & 0.501 \\
\hline $\mathrm{HF}$ & $14.7(650)$ & $18.8(87)$ & 0.025 \\
\hline NYHA class $3 / 4$ & $9.7(426)$ & $15.3(71)$ & $<0.001$ \\
\hline Cardiogenic shock & $7.3(322)$ & $10.3(48)$ & 0.021 \\
\hline CPA & 4.5 (197) & 7.8 (36) & 0.003 \\
\hline
\end{tabular}

$\mathrm{RD}$, right dominant; $\mathrm{Co}$, co-dominant; $\mathrm{LD}$, left dominant; $\mathrm{BMI}$, body mass index; $\mathrm{DM}$, diabetes mellitus; CVD, cerebrovascular disease; COPD, chronic obstructive pulmonary disease; $C K D$, chronic kidney disease; $P A D$, peripheral artery disease; $\mathrm{MI}$, myocardial infarction; $\mathrm{HF}$, heart failure; $\mathrm{PCl}$, percutaneous coronary intervention; $C A B G$, coronary artery bypass grafting; CCS, Canadian Cardiovascular Society; NYHA, New York Heart Association; CPA, cardiac pulmonary arrest.

doi:10.1371/journal.pone.0072672.t001
The majority of the clinical variables in the JCD were defined according to the National Cardiovascular Data Registry (NCDR), sponsored by the American College of Cardiology, to conduct comparative research and determine the factors that lead to disparities in PCI management. NCDR is a large PCI registry system with over 1,000,000 entries of ischemic heart disease and over 500,000 PCI entries collected from more than 500 institutions in the USA $[8,9]$.

Cardiogenic shock was defined as a sustained ( $>30$ minutes) episode of systolic blood pressure $<90 \mathrm{~mm} \mathrm{Hg}$, and/or a cardiac index of $<2.2 \mathrm{~L} / \mathrm{min} / \mathrm{m}^{2}$ determined to be secondary to cardiac dysfunction, and/or the requirement for parenteral inotropic or vasopressor agents or mechanical support (e.g., intra-aortic balloon pump [IABP], extracorporeal circulation, ventricular assist devices) to maintain blood pressure and a cardiac index above the levels specified. Heart failure was defined as physician documentation or reported clinical symptoms of heart failure, such as unusual dyspnea on light exertion, recurrent dyspnea occurring in the supine position, fluid retention; or the description of rales, jugular venous distension, or pulmonary edema on physical examination; or pulmonary edema evident in chest radiographs and presumed to be associated with cardiac dysfunction. A low ejection fraction, without clinical evidence of heart failure, did not qualify as heart failure. Cardiopulmonary arrest was defined as cardiac arrest and/or respiratory arrest before the PCI procedure.

PCI for urgent therapy was defined as a procedure performed on an inpatient basis because of significant concerns regarding a risk of ischemia, infarction, and/or death. However, urgent PCI was also performed on outpatients or emergency department

Table 2. Procedural information for each group.

\begin{tabular}{|c|c|c|c|}
\hline & $\begin{array}{l}\text { RD+Co \% } \\
(n=4409)\end{array}$ & $\begin{array}{l}\text { LD } \% \\
(n=464)\end{array}$ & $P$ Value \\
\hline \multicolumn{4}{|l|}{ Coronary status } \\
\hline STEMI & $48.2(2125)$ & $45.0(209)$ & 0.204 \\
\hline NSTEMI & $15.7(692)$ & $18.5(86)$ & 0.125 \\
\hline UA & 36.1 (1592) & 36.4 (169) & 0.919 \\
\hline 2-Vessel disease & $42.9(1890)$ & $46.1(214)$ & 0.184 \\
\hline 3-Vessel disease & 24.7 (1088) & $22.2(103)$ & 0.256 \\
\hline LMT stenosis & $9.3(410)$ & $6.9(32)$ & 0.089 \\
\hline \multicolumn{4}{|l|}{$\mathrm{PCl}$ indication } \\
\hline Emergent therapy & 48.3 (2129) & $49.1(228)$ & 0.733 \\
\hline Salvage therapy & $3.5(154)$ & $6.7(31)$ & 0.002 \\
\hline \multicolumn{4}{|l|}{ Stent Implantation } \\
\hline DES & $54.1(2387)$ & $55.8(259)$ & 0.493 \\
\hline BMS & $33.9(1493)$ & $30.8(143)$ & 0.197 \\
\hline Balloon angioplasty & 7.1 (314) & 8.4 (39) & 0.301 \\
\hline \multicolumn{4}{|l|}{ Puncture site } \\
\hline Radial artery & $23.9(1053)$ & $22.0(102)$ & 0.389 \\
\hline Femoral artery & $74.2(3273)$ & 76.7 (356) & 0.263 \\
\hline \multicolumn{4}{|l|}{ IABP insertion } \\
\hline Before PCI & $2.9(126)$ & $2.8(13)$ & 0.945 \\
\hline During/after $\mathrm{PCl}$ & $10.2(449)$ & $13.8(64)$ & 0.016 \\
\hline
\end{tabular}

$\mathrm{RD}$, right dominant; Co, co-dominant; LD, left dominant; STEMI, ST elevation myocardial infarction; NSTEMI, non-ST elevation myocardial infarction; UA unstable angina; LMT, left main trunk; $\mathrm{PCl}$, percutaneous coronary intervention; DES, drug eluting stent; BMS, bare metal stent; IABP, intra-aortic balloon pump. doi:10.1371/journal.pone.0072672.t002 
Table 3. In-hospital outcome/complications in each group.

\begin{tabular}{|c|c|c|c|}
\hline & RD+Co \% $(n=4409)$ & LD \% $(n=464)$ & $P$ Value \\
\hline All-cause death & $4.1(182)$ & $7.8(36)$ & 0.001 \\
\hline HF/shock/death & 7.9 (349) & $11.4(53)$ & 0.013 \\
\hline TIMI flow grade $<3$ & $6.2(273)$ & $6.7(31)$ & 0.686 \\
\hline $\mathrm{HB},>3 \mathrm{~g} / \mathrm{dL}$ decrease & $14.0(555)$ & $13.7(57)$ & 0.941 \\
\hline CIN & $19.6(864)$ & $87(18.8)$ & 0.712 \\
\hline Any complications & $13.6(600)$ & $15.1(70)$ & 0.395 \\
\hline Dissection & $1.5(52)$ & $1.1(5)$ & 1.000 \\
\hline Perforation & $0.7(33)$ & $0.9(4)$ & 0.776 \\
\hline MI & $2.1(92)$ & $2.4(11)$ & 0.613 \\
\hline $\mathrm{HF}$ & $3.3(146)$ & $4.5(21)$ & 0.179 \\
\hline Shock & $3.2(142)$ & $3.9(18)$ & 0.413 \\
\hline Dialysis & $1.8(79)$ & $0.9(4)$ & 0.184 \\
\hline Stroke & $0.7(31)$ & $0.4(2)$ & 0.765 \\
\hline $\mathrm{ICH}$ & $0.1(4)$ & $0.0(0)$ & 1.000 \\
\hline Transfusion & $3.7(161)$ & $4.3(20)$ & 0.440 \\
\hline Bleeding & 4.1 (179) & $5.8(27)$ & 0.088 \\
\hline Puncture site bleeding & $g 1.4(61)$ & $2.4(11)$ & 0.103 \\
\hline Hematoma & $1.0(43)$ & $0.6(3)$ & 0.620 \\
\hline Retroperitoneal & $0.1(4)$ & $0.2(1)$ & 0.394 \\
\hline Gastrointestinal & $0.6(28)$ & $1.5(7)$ & 0.044 \\
\hline Genitourinary & $0.3(12)$ & $0.6(3)$ & 0.166 \\
\hline Other & $1.5(68)$ & $1.7(8)$ & 0.694 \\
\hline
\end{tabular}

$\mathrm{RD}$, right dominant; Co, co-dominant; LD, left dominant; TIMI, thrombolysis in myocardial infarction; $\mathrm{HF}$, heart failure; $\mathrm{Hb}$, hemoglobin; $\mathrm{CIN}$, contrast induced nephritis; $\mathrm{Ml}$, myocardial infarction; $\mathrm{ICH}$, intracranial hemorrhage.

doi:10.1371/journal.pone.0072672.t003

patients when a cardiac catheterization was required and warranted an admission, based on the clinical presentation. PCI for emergent therapy was defined as a procedure that is performed as soon as possible because of substantial concerns that ongoing ischemia and/or infarction could lead to death. The phrase "as soon as possible" referred to a situation wherein a patient has a clinical presentation of sufficient gravity that a scheduled case would be canceled to perform this procedure. PCI for salvage therapy was defined as a procedure that was performed as a last resort; in such cases, the patient was in cardiogenic shock at the start of the procedure or within the 10 minutes prior to the start of the procedure, and the patient had also received chest compressions for a total of at least $60 \mathrm{~s}$ or had been on unanticipated extracorporeal circulatory support (e.g., extracorporeal membrane oxygenation, cardiopulmonary support).

\section{Information Disclosure}

Before the launch of the JCD, information on the objectives of the study, its social significance, and an abstract were provided to register this clinical trial with the University Hospital Medical Information Network. This Network is recognized by the International Committee of Medical Journal Editors as an "acceptable registry," according to a statement issued in September 2004 (UMIN R000005598).

\section{JCD Participants}

Major teaching hospitals within the metropolitan Tokyo area were selected for the pilot phase of this study, and the study
Table 4. Multivariate logistic regression analysis on inhospital mortality.

\begin{tabular}{llll}
\hline & OR & $\mathbf{9 5 \%}$ Cl & $P$ Value \\
\hline Left dominance & 1.75 & $1.06-2.89$ & 0.030 \\
Age, $\geq 80$ & 3.64 & $2.40-5.53$ & $<0.001$ \\
Age $70-79$ & 1.61 & $1.08-2.41$ & 0.02 \\
BMI $\geq 30$ & 2.23 & $1.20-4.46$ & 0.023 \\
CKD stage $\geq 3$ & 3.48 & $2.33-5.20$ & $<0.001$ \\
CCS class 4 angina & 2.11 & $1.12-3.99$ & 0.021 \\
STEMI & 4.59 & $2.16-9.74$ & $<0.001$ \\
NSTEMI & 3.58 & $1.68-7.65$ & 0.001 \\
Cardiogenic shock & 2.79 & $1.69-4.61$ & $<0.001$ \\
HF & 2.54 & $1.73-3.71$ & $<0.001$ \\
CPA & 2.42 & $1.21-4.85$ & 0.012 \\
LMT stenosis & 1.85 & $1.15-2.97$ & 0.011 \\
\hline
\end{tabular}

OR, odds ratio; $\mathrm{Cl}$, confidence interval; $\mathrm{BMI}$, body mass index; $\mathrm{CKD}$, chronic kidney disease; CCS, Canadian Cardiovascular Society; STEMI, ST elevation myocardial infarction; NSTEMI, non-ST elevation myocardial infarction; HF, heart failure; CPA, cardiac pulmonary arrest; LMT, left main trunk.

doi:10.1371/journal.pone.0072672.t004

protocol was approved by the institutional review board (IRB) committee at each site. In this registry, the data were collected

Table 5. In-hospital outcomes/complications in patients with cardiogenic shock and/or cardiopulmonary arrest.

\begin{tabular}{llll}
\hline & & & \\
\hline & RD+Co \% $(\mathbf{n = 3 7 1 )}$ & LD \% (n= 54) & $P$ Value \\
\hline All-cause death & $27.2(101)$ & $38.9(21)$ & 0.106 \\
HF/shock/death & $36.1(134)$ & $46.3(25)$ & 0.176 \\
TIMI flow grade $<3$ & $14.6(54)$ & $13.0(7)$ & 1.000 \\
Bleeding & $12.1(45)$ & $24.1(13)$ & 0.031 \\
CIN & $31.8(118)$ & $33.3(18)$ & 0.876 \\
\hline
\end{tabular}

$\mathrm{RD}$, right dominant; Co, co-dominant; LD, left dominant; HF, heart failure; TIMI, thrombolysis in myocardial infarction; $\mathrm{Hb}$, hemoglobin; $\mathrm{CIN}$, contrast induced nephritis.

doi:10.1371/journal.pone.0072672.t005

since September 2008 from the 14 Japanese hospitals participating in the Japanese Cardiovascular Database (JCD) [10-13]. All patients aged $>18$ years undergoing PCI or an acetylcholine challenge test in these hospitals were enrolled.

\section{Procedures and Data Collection}

In this study, all ACS patients who underwent PCI were included. We did not include those who underwent acetylcholine challenge tests in our analysis since, typically, patients undergo this challenge test only if they do not have obstructive lesions and vasospastic angina is suspected [14,15].

Data were analyzed from the 4873 patients undergoing PCI for ACS at one of the 14 Japanese hospitals participating in the JCDKeio Interhospital Cardiology Study (KICS) between September 2008 and April 2013.

ACS is used to describe ST-elevation myocardial infarction (STEMI), non ST-elevation myocardial infarction (NSTEMI), and unstable angina (UA). STEMI was defined as myocardial 
Table 6. In-hospital outcomes/complications in patients with left main trunk stenosis.

\begin{tabular}{llll}
\hline & & & \\
\hline & RD+Co \% $(\mathbf{n}=\mathbf{4 1 0})$ & LD \% (n= 32) & P Value \\
\hline All-cause death & $10.7(44)$ & $18.8(6)$ & 0.239 \\
HF/shock/death & $17.8(73)$ & $18.8(6)$ & 0.814 \\
TIMl flow grade $<3$ & $9.0(37)$ & $12.5(4)$ & 0.523 \\
Bleeding & $7.8(32)$ & $3.1(1)$ & 0.496 \\
CIN & $26.1(107)$ & $15.6(5)$ & 0.213 \\
\hline
\end{tabular}

$\mathrm{RD}$, right dominant; $\mathrm{Co}$, co-dominant; LD, left dominant; HF, heart failure; TIMI, thrombolysis in myocardial infarction; $\mathrm{Hb}$, hemoglobin; $\mathrm{CIN}$, contrast induced nephritis.

doi:10.1371/journal.pone.0072672.t006

infarction with ST elevation. These elements are equivalent to the definitions provided in the associated guidelines $[8,9,16]$. When anterior lead-ST depression was documented as 'STEMI equivalent' by the attending cardiologist, they were also coded as STEMI. On the other hand, if there was no written documentation of STEMI in cases of V1-3 ST depression, those cases were coded NSTEMI.

Patients included in the study were divided into 2 groups, based on a diagnostic coronary angiogram performed prior to their PCI; patients with right- or co-dominant anatomies were placed into the RD group and those with left-dominant anatomies were included in the LD group. RD and co-dominant patients were placed into the same group because these patients have been demonstrated to have similar prognoses [3]. Moreover, the hypothesis that PDAs, arising from the right coronary artery, may serve as a back-up blood supply in individuals with normal anatomy, would indicate that individuals with a co-dominant anatomy would be expected to derive similar benefits to those with RD anatomy. LD anatomy was defined as one in which the PDA originated from the LCX. $\mathrm{RD}$ anatomy was defined as the PDA originating from the RCA. Co-dominant anatomy was defined when only the PDA originated from the RCA and the RCA did not give rise to posterolateral branches, in combination with a large posterolateral branch originating from the LCX reaching near the posterior interventricular groove [6]. The dominance of each patient was documented by the treating cardiologist and failure to document dominance information was detected by the clinical coordinator and its input was mandated by the site data manager.

This research was supported by a grant from the Ministry of Education, Culture, Sports, Science, and Technology, Japan (KAKENHI No. 21790751). The JCD Steering Committee was responsible for overall study guidance, including the study protocol, data analyses, and interpretation of results. The Department of Healthcare Quality Assessment at Tokyo University independently managed the database. The KICS Committee managed the participating sites and provided monthly on-site monitoring services to assure data accuracy and completeness throughout the study. During the planning, implementation, and reporting of this study, there were no issues such as conflicts of interest, conflicts of responsibility, or intellectual property right concerns.

The study endpoints included in-hospital mortality, heart failure, cardiogenic shock, and other complications. Complications were defined as all complications, including severe dissection or coronary perforation, myocardial infarction after PCI, contrastinduced nephritis, cardiogenic shock or heart failure, cerebral bleeding or stroke, and bleeding complications. Bleeding compli- cations in this registry were defined as those requiring transfusion, prolonging hospital stay, and/or causing a decrease in hemoglobin of $>3.0 \mathrm{~g} / \mathrm{dL}$ [17]. Further, bleeding complications were subdivided into puncture-site bleeding, retroperitoneal bleeding, gastrointestinal bleeding, genitourinary bleeding, or other bleeding.

\section{Data Analyses}

Continuous variables are expressed as means and standard deviations (SD); categorical variables are expressed as percentages. Continuous variables were compared using Student's $t$-test, and differences between categorical variables were examined using a chi-squared test. A multiple logistic regression analysis was performed to determine the independent predictors of in-hospital mortality. Factors, at admission, that were evaluated in the multivariate model were age, $70-79$ years; age, $\geq 80$ years; body mass index $(\mathrm{BMI}) \geq 30$; presence of chronic kidney disease stage $\geq 3$; presence of chronic obstructive pulmonary disease; presence of Canadian Cardiovascular Society class 4 angina; dialysis dependence; presence of heart failure; cardiogenic shock; cardiopulmonary arrest; left main trunk stenosis; STEMI; NSTEMI; PCI for emergent therapy; PCI for salvage therapy; and LD anatomy. All statistical calculations and analyses were performed using SPSS version 15 (SPSS, Chicago, IL, USA). $P$-values of $<0.05$ were considered statistically significant.

\section{Results}

A total of 4873 ACS patients undergoing PCI were assessed. The average age of the patients was $67.6 \pm 11.8$ years and 1081 $(22.2 \%)$ patients were female. The numbers of STEMI, NSTEMI, and UA patients were $2334(47.9 \%), 778$ (16.0\%), and 1761 $(36.1 \%)$, respectively. The LD group was comprised of 464 patients (9.5\%), and there were 4409 patients (90.5\%) in the RD group. Among 4873 ACS patients, 639 (12.4\%) had co-dominant anatomy.

\section{Baseline Characteristics}

The baseline clinical characteristics of the 4873 patients in both groups are summarized in Table 1. There were no major differences in the coronary risk factors, BMI, comorbidities (cerebrovascular disease, chronic obstructive pulmonary disease, presence of chronic kidney disease stage $\geq 3$, hemodialysis, peripheral artery disease), or past history (prior myocardial infarction, prior heart failure, prior PCI, prior coronary artery bypass grafting). The numbers of patients presenting with symptoms of heart failure, cardiogenic shock, or cardiopulmonary arrest were significantly higher in the LD group than in the RD group (heart failure: $650 \mathrm{RD}$ patients [14.7\%] vs. $87 \mathrm{LD}$ patients [18.8\%], $P=0.025$; cardiogenic shock: $322 \mathrm{RD}$ patients [7.3\%] vs. $48 \mathrm{LD}$ patients [10.3\%], $P=0.021$; and cardiopulmonary arrest: $197 \mathrm{RD}$ patients $[4.5 \%$ vs. $36 \mathrm{LD}$ patients [7.8\%], $P=0.003$ ) (Table 1).

\section{Angiographical Data and Procedural Information}

The angiographical and procedural data are listed in Table 2. The number of patients with STEMI, NSTEMI, and UA were similar between the groups. There were no statistically significant differences in the proportions of drug eluting stents implanted (2387 RD patients $[54.1 \%]$ vs. $259 \mathrm{LD}$ patients $[55.8 \%]$, $P=0.493)$, nor in the proportions of bare metal stents implanted (1493 RD patients [33.9\%] vs. $143 \mathrm{LD}$ patients [30.8\%], $P=0.197)$.

The proportions of IABPs used were analyzed in both groups. The numbers of IABP insertions before PCI procedures were 
similar in both groups (126 RD patients [2.9\%] vs. $13 \mathrm{LD}$ patients $[2.8 \%], P=0.945)$. However, the number of IABP insertions during and after PCI procedures was significantly higher in the LD group than in the RD group (449 RD patients [10.2\%] vs. $64 \mathrm{LD}$ patients [13.8\%], $P=0.016)$.

\section{In-hospital Outcomes/Complications}

Table 3 shows the overall in-hospital outcomes and complications for the 2 groups of patients. The in-hospital mortality rate was significantly higher in the LD group compared to the RD group (182 RD patients [4.1\%] vs. $36 \mathrm{LD}$ patients [7.8\%], $P=0.001$ ) (Table 3). The combined rates of heart failure, cardiogenic shock, and death were also significantly higher in the LD group than in the RD group (349 RD patients [7.9\%] vs. $53 \mathrm{LD}$ patients [11.4\%], $P=0.013)$. As listed in Table 3, the rates of thrombolysis in myocardial infarction (TIMI) flow that were $<$ grade 3 , the rates of hemoglobin decreases of $>3 \mathrm{~g} / \mathrm{dL}$, the rates of contrast-induced nephritis, and the rates of other complications, including bleeding complications, were similar for the 2 groups. Only gastrointestinal bleeding complications were significantly different between the groups $(28 \mathrm{RD}$ patients $[0.6 \%]$ vs. $7 \mathrm{LD}$ patients $[1.5 \%], P=0.044)$.

Multivariate logistic regression analysis showed that $\mathrm{LD}$ anatomy was one of the independent predictors for in-hospital mortality (odds ratio, 1.75; 95\% confidence interval, 1.06-2.89; $P=0.030)$. Cardiogenic shock, heart failure, and cardiopulmonary arrest were also independent predictors for in-hospital mortality (Table 4).

\section{In-hospital Outcomes in Selected Subgroups}

Tables 5 and 6 show the results from the data analysis conducted in selected subgroups of high-risk patients. LD patients tended to have higher rates of in-hospital mortality for patients with cardiogenic shock and/or cardiopulmonary arrest (101 RD patients [27.2\%] vs. $21 \mathrm{LD}$ patients [38.9\%], $P=0.106$ ) (Table 5), or left main trunk stenosis (44 RD patients [10.7\%] vs. $6 \mathrm{LD}$ patients [18.8\%], $P=0.239$ ) (Table 6).

\section{Discussion}

The present study concluded that coronary dominance affected in-hospital outcomes. LD patients had significantly worse inhospital outcomes compared with RD patients and LD anatomy was an independent predictor of in-hospital mortality in the multivariate analysis. The significance of coronary dominance should be taken into consideration when treating ACS patients with PCI. The absence of the protective effects of a double supply to the myocardium and technical challenges caused by the anatomy may be particularly important in these patients.

Previous studies also have shown that LD patients with coronary artery disease may have worse prognoses than RD patients with coronary artery disease. Goldberg et al. demonstrated that LD is a significant and independent predictor of increased long-term mortality in patients with ACS [3]. However, in their study, the revascularization rate (by PCI or coronary artery bypass graft [CABG]) was approximately $60 \%$ and the number of patients with stent implantation was not reported. In the present study, PCI was attempted in all patients.

The higher in-hospital mortality associated with LD patients led to the hypothesis that the RCA serves as a back-up supply in patients with RD anatomy, providing a measure of protection for the myocardium in ACS patients. According to one study [18], patients with acute occlusion of the LCX, presenting with NSTEMI, had better outcomes than did those with STEMI.
The analysis indicated that patients with RD anatomies were more likely to have NSTEMI. In concordance with our study, the authors asserted that RD may confer a protective effect in cases of acute occlusion of the LCX, minimizing the infarct size. The data also revealed that smaller infarct sizes and higher likelihoods of RD were associated with NSTEMI patients, supporting their conclusion.

In general, a dominant LCX has several acute angles in its course, including at its origin and at its distal end where it becomes the PDA. These acute angles lead to turbulence and shear stress during blood flow that, in turn, may enhance thrombus formation and platelet activity $[19,20]$. The acute angles, and resultant turbulence and shear stress, also contribute to the difficulty of LCX interventions. Yip et al. concluded that LD is related to unsuccessful reperfusion and to a higher 30-day mortality rate in LCX infarct-related acute myocardial infarction (MI) patients [21]. In addition, Auriti et al. showed that coronary flow reserve was more impaired in LCX than LAD just after Y-graft intervention, which was a graft formed by the left internal mammary artery (LIMA) connected to the LAD and by a free right internal mammary artery (RIMA) connected to LIMA and a marginal artery of the LCX [22]. Clinically, a recent study showed that the 30-day prognostic outcome was less favorable in LCXrelated acute inferior MI compared to RCA-related acute inferior MI [23]. The reasons for this difference were suggested to include higher peak levels of creatine kinase-MB isoenzyme, lower left ventricular ejection fractions, and the higher numbers of advanced congestive heart failure that were observed in LCX-related acute inferior MIs. Lower rates of stent implantation and collateral circulation might also contribute to the worse outcomes. The study data did not indicate the manner in which dominance might affect mortality; Kim concluded patients with an occluded LCX presented with less ST elevation and primary PCI [24]. Multivariate analysis showed that primary PCI decreased the hospital mortality for patients with occluded coronary arteries. For these reasons, we suspect that more flow disturbances are induced, leading to more adverse effects on hemodynamics during PCI, in patients with PCI of a dominant LCX.

Larger infarct size is another reason affecting in-hospital mortality for LD patients. IABPs might be effective for limiting infarct sizes in such cases. Pierrakos et al. showed that during reperfusion, IABPs increased coronary blood flow, and effectively limited infarct size [25]. Our study showed that the proportion of IABP insertions during and after PCI procedures was significantly higher in the LD group than in the RD group. We suspect that the LD group tended to have hemodynamic instability more frequently than the RD group during the procedure because of the single coronary supply. Moreover, to overcome the difficulty for intervening LD LCX arteries with PCI, CABG would be considered. However, further prospective study will be necessary.

\section{Study Limitations}

Several limitations may exist in this study. First, this study was an analysis of a multicenter cohort study rather than an observational, non-randomized trial. Second, the study population was of limited size, compared to the number of sites included in our registry. Third, we did not analyze whether the target vessel lesions compromised PDA function. Lastly, all candidates underwent PCI procedures. Some patients with critical conditions, such as cardiogenic shock or left main trunk stenosis, are more favorable candidates for CABG than for PCI and these patients, who underwent CABG instead of PCI, were excluded. Therefore, we do not have data indicating which treatment was better for these patients. 


\section{Conclusions}

LD patients had higher in-hospital mortality compared with RD patients in a population with ACS patients; LD anatomy was an independent predictor for in-hospital mortality in the multivariate analysis in this Japanese, real-world, multicenter study. A single coronary supply, typical of patients with an LD anatomy, should be recognized as a high-risk feature.

\section{Acknowledgments}

We appreciate all the investigators, clinical coordinators, and institutions involved in the JCD-KICS study.

Investigators: Yohei Numasawa, Toshiki Kuno, Toshiyuki Takahashi (Ashikaga Red Cross Hospital), Yutaka Okada (Eiju General Hospital), Soushin Inoue, Iwao Nakamura (Hino Municipal Hospital), Shunsuke Takagi, Takashi Matsubara (Hiratsuka City Hospital), Masashi Takahashi, Keishu Li, Koichiro Sueyoshi (Kawasaki City Municipal Hospital), Taku Inohara, Atsushi Anzai, Kentaro Hayashida, Takashi Kawakami, Hideaki Kanazawa, Takahide Arai, Shunsuke Yuasa, Jun Fujita, Yuichiro Maekawa, Akio Kawamura (Keio University School of Medicine),

\section{References}

1. Gorlin R (1976) Coronary anatomy. Major Probl Intern Med 11: 40-58.

2. Bazzocchi G, Romagnoli A, Sperandio M, Simonetti G (2011) Evaluation with 64-slice CT of the prevalence of coronary artery variants and congenital anomalies: a retrospective study of 3,236 patients. Radiol Med 116: 675-89.

3. Goldberg A, Southern DA, Galbraith PD, Traboulsi M, Knudtson ML, et al (2007) Coronary dominance and prognosis of patients with acute coronary syndrome. Am Heart J 154: 1116-22.

4. Eren S, Bayram E, Fil F, Koplay M, Sirvanci M, et al (2008) An investigation of the association between coronary artery dominance and coronary artery variations with coronary arterial disease by multidetector computed tomographic coronary angiography. J Comput Assist Tomogr 32: 929-33.

5. Vasheghani-Farahani A, Kassaian SE, Yaminisharif A, Davoodi G, Salarifar M, et al (2008) The association between coronary arterial dominancy and extent of coronary artery disease in angiography and paraclinical studies. Clin Anat 21: 519-23.

6. Veltman CE, de Graaf FR, Schuijf JD, van Werkhoven JM, Jukema JW, et al (2012) Prognostic value of coronary vessel dominance in relation to significant coronary artery disease determined with non-invasive computed tomography coronary angiography. Eur Heart J 33: 1367-77.

7. Goto M, Kohsaka S, Lee VV, Elayda MA, Aoki N, et al (2011) Risk stratification of coronary revascularization patients by using clinical and angiographic data. J Cardiovasc Surg (Torino) 52: 863-71.

8. Roe MT, Messenger JC, Weintraub WS, Cannon CP, Fonarow GC, et al (2010) Treatments, trends, and outcomes of acute myocardial infarction and percutaneous coronary intervention. J Am Coll Cardiol 56: 254-63.

9. Anderson HV, Shaw RE, Brindis RG, McKay CR, Klein LW, et al (2007) Riskadjusted mortality analysis of percutaneous coronary interventions by American College of Cardiology/American Heart Association guidelines recommendations. Am J Cardiol 99: 189-96.

10. Mogi S, Kohsaka S, Miyata H, Kawamura A, Noma S, et al (2011) Comparison between working day and holiday acute coronary syndrome presentation. Int J Cardiol 153: 85-7.

11. Numasawa Y, Kohsaka S, Mivata H, Kawamura A, Noma S, et al (2013) Safety of transradial approach for percutaneous coronary intervention in relation to body mass index: a report from a Japanese multicenter registry. Cardiovasc Interv Ther 28: 148-56.

12. Arai T, Yuasa S, Miyata H, Kawamura A, Maekawa Y, et al (2012) Incidence of periprocedural myocardial infarction and cardiac biomarker testing after percutaneous coronary intervention in Japan: results from a multicenter registry. Heart Vessels. [Epub ahead of print].

13. Numasawa Y, Kohsaka S, Miyata H, Kawamura A, Noma S, et al (2013) Use of Thrombolysis in Myocardial Infarction Risk Score to predict bleeding complications in patients with unstable angina and non-ST elevation myocardial infarction undergoing percutaneous coronary intervention. Cardiovasc Interv Ther. [Epub ahead of print].
Masahiro Suzuki (National Hospital Organization Saitama National Hospital) Yukinori Ikegami, Yukihiko Momiyama (National Hospital Organization Tokyo Medical Center), Ayaka Endo, Taku Hasegawa, Toshiyuki Takahashi, Susumu Nakagawa (Saiseikai Central Hospital), Takashi Yagi, Kenichiro Shimoji, Sonhan Yun, Takeshi Onitsuka, Shigetaka Noma (Saiseikai Utsunomiya Hospital), Shiro Ishikawa (Saitama City Hospital), Atsushi Mizuno, Yutaro Nishi (St Luke's International Hospital Heart Center), Masaru Shibata, Takashi Koyama (Tachikawa Kyosai Hospital), Kimi Koide, Yoshinori Mano, Takahiro Oki (Tokyo Dental College Ichikawa General Hospital), Daisuke Shinmura, Kouji Negishi (Yokohama Municipal Citizens Hospital).

Clinical Coordinators: Junko Susa, Ayano Ishikawa, Hiroaki Nagayama, Miho Umemura, Itsuka Saito, Kiri Shimauchi, Mizue Sato, Shino Nakamura, Makiko Tsukamoto and Ikuko Ueda.

\section{Author Contributions}

Conceived and designed the experiments: TK YN SK. Analyzed the data: HM. Wrote the paper: TK. Revised the manuscript: YN TT KS TO KN AK SK KF. Created the database: HM SK.

14. Ong P, Athanasiadis A, Borgulya G, Mahrholdt H, Kaski JC, et al (2012) High prevalence of a pathological response to acetylcholine testing in patients with stable angina pectoris and unobstructed coronary arteries. The ACOVA Study (Abnormal COronary VAsomotion in patients with stable angina and unobstructed coronary arteries). J Am Coll Cardiol 59: 655-62.

15. Ong P, Athanasiadis A, Borgulya G, Voehringer M, Sechtem U (2011) 3-year follow-up of patients with coronary artery spasm as cause of acute coronary syndrome: the CASPAR (coronary artery spasm in patients with acute coronary syndrome) study follow-up. J Am Coll Cardiol 57: 147-52.

16. Anderson JL, Adams CD, Antman EM, Bridges CR, Califf RM, et al (2011) 2011 ACCF/AHA Focused Update Incorporated Into the ACC/AHA 2007 Guidelines for the Management of Patients With Unstable Angina/Non-STElevation Myocardial Infarction: a report of the American College of Cardiology Foundation/American Heart Association Task Force on Practice Guidelines. Circulation 123: e426-579.

17. Mehta SK, Frutkin AD, Lindsey JB, House JA, Spertus JA, et al (2009) Bleeding in patients undergoing percutaneous coronary intervention: the development of a clinical risk algorithm from the National Cardiovascular Data Registry. Circ Cardiovasc Interv 2: 222-9.

18. Stribling WK, Kontos MC, Abbate A, Cooke R, Vetrovec GW, et al (2011) Clinical outcomes in patients with acute left circumflex/obtuse marginal occlusion presenting with myocardial infarction. J Interv Cardiol 24: 27-33.

19. Yu SK, Latour JG, Marchandise B, Bois M (1979) Shear stress-induced changes in platelet reactivity. Thromb Haemost 40: 551-60.

20. Smith RL, Blick EF, Coalson J, Stein PD (1972) Thrombus production by turbulence. J Appl Physiol 32: 261-4.

21. Yip HK, Wu CJ, Fu M, Yeh KH, Yu TH, et al (2002) Clinical features and outcome of patients with direct percutaneous coronary intervention for acute myocardial infarction resulting from left circumflex artery occlusion. Chest 122: 2068-74.

22. Auriti A, Loiaconi V, Pristipino C, Leonardi Cattolica FS, Cini R, et al (2010) Recovery of distal coronary flow reserve in LAD and LCx after Y-Graft intervention assessed by transthoracic echocardiography. Cardiovasc Ultrasound 8: 34

23. Chen YL, Hang CL, Fang HY, Tsai TH, Sun CK, et al (2011) Comparison of prognostic outcome between left circumflex artery-related and right coronary artery-related acute inferior wall myocardial infarction undergoing primary percutaneous coronary intervention. Clin Cardiol 34: 249-53.

24. Kim SS, Choi HS, Jeong MH, Cho JG, Ahn YK, et al (2011) Clinical outcomes of acute myocardial infarction with occluded left circumflex artery. J Cardiol 57: 290-6.

25. Pierrakos CN, Bonios MJ, Drakos SG, Charitos EI, Tsolakis EJ, et al (2011) Mechanical assistance by intra-aortic balloon pump counterpulsation during reperfusion increases coronary blood flow and mitigates the no-reflow phenomenon: an experimental study. Artif Organs 35: 867-74. 